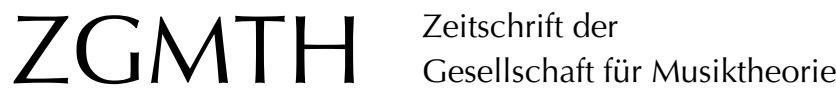

Diergarten, Felix (2011): VII. European Music Analysis Conference (Euromac), Rom, 29. September bis 2. Oktober 2011. ZGMTH 8/2, 369-371.

https://doi.org/10.31751/646

(C) 2011 Felix Diergarten

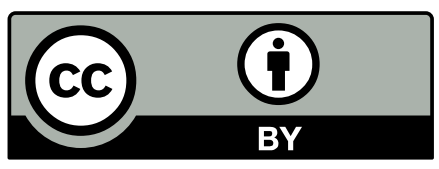

Dieser Text erscheint im Open Access und ist lizenziert unter einer Creative Commons Namensnennung 4.0 International Lizenz.

This is an open access article licensed under a

Creative Commons Attribution 4.0 International License.

veröffentlicht / first published: 17/11/2011

zuletzt geändert / last updated: 11/11/2011 


\section{European Music Analysis Conference (Euromac), Rom, 29. September bis 2. Oktober 2011}

„Man müsste mit tausend Griffeln schreiben, was soll hier eine Feder! « Mit diesem Stoßseufzer entledigte sich ein bekannter deutscher Italienreisender des 18. Jahrhunderts elegant der Pflicht, akkurat und detailgetreu zu benennen, was ihm sein erster römischer Aufenthalt täglich aufgab. Mit diesen Worten entledigt sich auch der Autor dieser Zeilen von vorne herein des Anspruchs, einen repräsentativen Querschnitt, geschweige denn eine umfassende Darstellung der VII. European Music Analysis Conference (kurz: Euromac) zu liefern, die vom 29. September bis 2. Oktober $2011 \mathrm{im}$ Conservatorio di Musica S. Cecilia in Rom stattfand. Bei einer insgesamt über 200 Darbietungen in sechs parallelen Sektionen umfassenden Konferenz ist es unter den Gesetzen von Raum und Zeit für eine einzelne Person möglich, etwa 50 dieser Darbietungen (also knapp ein Viertel) zu besuchen. Angesichts der Tatsache jedoch, dass sich die Konferenz in Fußnähe von spanischer Treppe, Piazza del Popolo und Villa Borghese abspielte, scheint es eher unwahrscheinlich, dass einer der Teilnehmer diese Zahl auch nur annähernd erreicht haben könnte - oder sollte sich der Eindruck merkwürdiger Menschenleere vielmehr durch die schier unendlichen Gänge und Treppenhäuser des Konservatoriums und ehemaligen Klostergebäudes erklären, in dessen heruntergekommener Grandezza sich die Teilnehmer verloren? Wie also über eine solche Konferenz berichten? Es bleibt mir nur die Flucht nach vorn in einen kurzgefassten Versuch, mir meinen eigenen Reim auf diese Großveranstaltung zu machen bzw. auf jenen Bruchteil, den ich davon erlebt habe.

Sowohl dem Programmbuch als auch der allen Teilnehmern ausgehändigten Stoffta- sche war eine Schenker-Grafik aufgedruckt, die von vorne herein mit einer im Jahre 2011 irgendwie trotzig anmutenden Geste signalisierte, wo zumindest für die federführenden Herren und Damen der analytische Hammer hängt: Hier wird smusic analysis` offenbar noch ganz wörtlich im ehemals angloamerikanischen Sinne unilateral verstanden, unangefochten von allen postschenkerianischen Diskussionen. Oder zumindest soll der Eindruck erweckt werden, dem sei so: Denn letztlich war nur eine einzige, vier Vorträge umfassende Sektion explizit Fragen der Schenker-Analyse gewidmet, die sich der zeitgleichen Konkurrenz der Sektionen "Liszt», "Schemata Theories», "Film Music», »Hermeneutics" und »Temporality« stellen musste. Das Konferenzprogramm zeigte also vielmehr eine fast einschüchternde Vielfalt von Themen und Methoden. Neben den bereits erwähnten standen unter anderem »Italian Opera», „Opera and Stage«, »Analysis and History«, "Analysis and Performance», „Oral Traditions", „Popular Music», »Post-tonal Form «, "20th-century Harmony", "Syntax and Metre ", "Tonal Theory«, »Music and Infancy", »Psychoanalytical Issues«, »Opera in France and Britain«, "Medieval and Renaissance Music» »Baroque Music«, »Romantic Form«, "Compositional Processes«, »Timbre and Texture« sowie Sektionen zu den Komponisten Corelli, Wagner, Debussy und Scelsi auf dem Programm.

Wem kämen angesichts einer so unübersichtlichen Situation nicht reflexartig gesellschafts- und fachpolitisch handliche Begriffe wie sPluralismus`, sMultivalenz`, ,Vielfalt der Zugänge etc. in den Sinn? Freilich kaschieren derartige Euphemismen häufig nur, dass 
sich die einzelnen Partikel dieser Vielfalt offensichtlich immer weniger unter einen Hut bringen lassen. So gesehen spiegelt das Kongressprogramm vor allem eine zunehmende Partikularisierung, in deren Konsequenz es liegt, dass die Vertreter der jeweiligen Partikel mitunter nur geringes Interesse an einem partikelübergreifendem Austausch zeigen zumindest wenn man unter >Austausch versteht, das über mal mehr, mal weniger respektvolle Kenntnisnahme hinausgeht. Gewiss: unter dem Aspekt einer methodischen Ausdifferenzierung und Professionalisierung der Teilbereiche lässt sich nicht erwarten, dass ein einzelner Forscher über spätmittelalterliche Hexachorde ebenso gewandt parliert wie über Zwölfton-Improvisation, Ennio Morricone und Gender-Studies. Trotzdem: Wer sich öffentlich zu einem facettenreichen Thema äußert, stellt sich unweigerlich dem Anspruch, fachlichen Mindeststandards zu genügen, die für dessen Teilbereiche gelten. Man müsste meinen, eine europäische Konferenz mit internationaler Ausstrahlung verfolge vor allem den Zweck, in diesem Sinne den Austausch sowohl zwischen den nationalen und lokalen Traditionen als auch zwischen den Subdisziplinen zu befördern und zu dokumentieren. Die römische Euromac jedoch legte offen, dass genau dieser Austausch nach wie vor kaum stattfindet. Ich erlaube mir, kurze Beispiele aus jenen Teilbereichen anzuführen, mit denen ich mich selbst einigermaßen professionell vertraut fühle, auch auf die Gefahr hin, voreingenommen zu sein und andere, wichtige Themen auszublenden.

Als symptomatisch für die Verweigerung eines echten fachlichen Austausches dürfte es gelten, wenn beispielsweise in einem Vortrag, der sich Aspekten der Renaissancemusik aus kommunikationstheoretischer Perspektive nähert, über historische Improvisationstechniken auf einem Niveau gesprochen, das den anwesenden Kennern dieser gründlich erforschten und praktisch erprobten Materie (vorsichtig ausgedrückt) die Stirn in Falten schlägt. Kaum weniger irritierten manche Beiträge zum "Satzmodell«-Diskurs, der in der deutschsprachigen Musiktheorie seit Jahren auf hohem Niveau und mit bemerkenswerter historischer und methodischer Differenzierung geführt wird, international dagegen auf einem basalen, über Gjerdingens Music in the Galant Style nicht substantiell hinausgehenden Niveau zu stagnieren scheint. Positive Gegenbeispiele boten die Beiträge zur jüngeren Formenlehre: Einem reichen europäischen Erfahrungsschatz traten auf diesem Gebiet seit den 90er Jahren die entschlossenen Statements der angloamerikanischen New Formenlehres zur Seite (und entgegen), die trotz der Kritik aus Europa offenbar auch diesseits des Ozeans mittlerweile breit rezipiert und nun in europäischen Beiträgen wiederum produktiv weitergedacht werden. In fruchtbarer Internationalität zeigte sich auch die kleine Gruppe der Partimentoforscher: Hier geht man (so mein Eindruck) nach einer aufregenden und aufgeregten Pionierzeit in eine Phase der nüchternen Professionalisierung über, in der etwa einzelne Manuskriptfamilien in ihrer Überlieferungssituation und Filiation oder lokale europäische Subtraditionen und Gegenentwürfe untersucht werden, was zu einem immer differenzierteren Bild musiktheoretischer Lehrtraditionen des 18. und 19. Jahrhunderts, gerade auch in ihrem heteronomen Nebeneinander, führen dürfte.

Die ungezwungene Atmosphäre, der prompte Pausenkaffee mit Pasticceria, das ansprechende social dinner und der römische Spätsommer: all dies trug wesentlich zu einem positiven Gesamteindruck bei. Getrübt wurde dieser allerdings durch einige Aspekte, auf die hinzuweisen ich mir verkneifen würde, wäre mir mein Eindruck nicht vielfach bestätigt worden: Unverhohlen kurzfristig zusammengeschusterte Präsentationen, ins Uferlose ausgreifende Monologe, die sich (statt der geforderten 20) weit über 30 Minuten hinstrecken, demonstrativ desinteressierte, unvorbereitete Sektionsmoderatoren, die nicht in der Lage oder nicht willens sind, den Programmverlauf mit einem gewissen Rest an Form anzukündigen geschweige denn zu regeln, und die sich weigern, selbst als Leiter einer mehrsprachigen Sektion eine andere Sprache als ihre eigene zu sprechen: das alles kann ich nicht mehr 
als charmante Ungezwungenheit oder professorale Weltvergessenheit belächeln. Nicht ernst genommen fühlt man sich auch, wenn bereits publizierte Texte ihre Zweit- oder Drittverwertung als Vortrag in geringfügig angepasster Form finden. Wenngleich man sich also vielleicht von dem Anspruch verabschieden muss, bei einer Konferenz dieser Größe müsse es deutliche übergeordnete Leitfragen geben, klare Trends, eine Handvoll für alle gemeinsam relevanter Themen oder gar eine allgemein akzeptierte Vorstellung davon, was unter »music analysis « zu verstehen sei - nicht verabschieden darf man sich jedenfalls bei aller Diversität von gemeinsamen und verbindlichen Qualitätsstandards für Form und Inhalt: Ohne diese würde der Sinn des Ganzen ernsthaft in Frage gestellt, und wie deren Einhaltung unter den gegebenen Voraussetzungen zu gewährleisten ist, dürfte ein wichtiges Thema auf dem Weg zur nächsten Euromac sein, die 2014 in Leuven stattfinden wird.

Felix Diergarten 